\title{
Evaluation of Efficacy and Toxicity of Helical Tomotherapy in the Treatment of Patients with Nasal NK/T-Cell Lymphoma
}

\author{
Baolin $\mathbf{Q u}^{1}{ }^{*}$, Xiao Zhao1, Lingling Meng1, Shouping Xu${ }^{1}$, Chuanbin Xie', Jack Yang² \\ ${ }^{1}$ Department of radiotherapy, PLA General Hospital, Beijing, China \\ ${ }^{2}$ Department of Radiation Oncology, Monmouth Medical Center, Long Branch, USA \\ Email: ${ }^{*}$ qubl6212@sina.com
}

Received 27 December 2014; accepted 14 January 2015; published 20 January 2015

Copyright (C) 2015 by authors and Scientific Research Publishing Inc.

This work is licensed under the Creative Commons Attribution International License (CC BY). http://creativecommons.org/licenses/by/4.0/

Open Access

\begin{abstract}
Purpose: Nasal lymphoma created dosimetric challenges in radiotherapy due to the complex anatomical structures. This study was to evaluate the efficacy and toxicity of helical Tomotherapy (HT) in the treatment of nasal NK/T-cell lymphoma (NKTCL) patients. Methods and Materials: Between August 2008 and April 2013, a total of 25 NKTCL patients were treated with HT in our department; Among them, three patients have not received chemotherapy, one patient has received concurrent chemo-radiation with CHOP plus L-ASP, two patients have received the sequential chemotherapy regimen following irradiation, and all the others have received 1 - 2 cycles of induction chemotherapy followed by irradiation and then with sequential chemotherapy. CHOP-L with 1 - 7 cycles (median: 4 cycles) was utilized as the main chemotherapy regimen. As for HT, the gross tumor volume (GTV) received target doses (TD) ranging from 50 to $56 \mathrm{~Gy}$ (median: $50 \mathrm{~Gy}$ ) at $2-2.78 \mathrm{~Gy}$ per fraction; and the clinical target volume (CTV) from 36 to $50 \mathrm{~Gy}$ (median: $40 \mathrm{~Gy}$ ) at 1.6 - 2 Gy per fraction. Results: For those patients who had received irradiation, thirteen achieved complete remission (CR), four partial responses (PR); four had progressive disease (PD), two were lost to follow-up, two died within one month after irradiation and were not followed. In 21 patients with follow-up records, the overall response (CR + PR) was $81.0 \%$ with the 3-year survival rate of 87.2\%, and the mean survival time was 52.8 months [95\% confidence interval (CI): $45.2-60.4$ months]. After radiotherapy the majority of patients had dry mouth and taste changes in varying degrees, and a small portion of patients had compromised hearing or vision functions. No brain injury symptoms occurred during radiation radiotherapy. Conclusions: As compared with conventional three-dimensional conformal radiotherapy (3D-CRT) and intensity modulated radiation therapy (IMRT) performed with HT, HT appears to have more favorable efficacy and toxicity profiles in the treatment of NKTCL. Further systematic and randomized clinical research is under investigation.
\end{abstract}

\footnotetext{
${ }^{*}$ Corresponding author.
} 


\section{Keywords}

\section{Tomotherapy, NK/T Cell Lymphoma, IMRT, Survival}

\section{Introduction}

Nasal NK/T-cell lymphoma (NKTCL) is an uncommon disease, but it is much more frequent in Asia and Latin American countries than in Western countries. NKTCL is classified as a special type of non-Hodgkin's lymphoma (NHL) with clinical staging [1]. Patients usually presented symptoms with necrotic changes in the nasal cavity. Young and middle-aged males are more likely to be affected, and the ratio of males to females being 2 to 4 factors to 1. In Asia, 67\% - 98\% of the patients were diagnosed with the disease at the localized stage IE-IIE, and regional lymph node and distant metastases are rare [2]. Although resistant to chemotherapy, the tumor is sensitive to irradiation with good responses. Radiotherapy was considered as the main treatment modality for early-stage NKTCL. However, patients had a poor prognosis if diagnosed with this disease at a late stage. Research work has shown that in comparison with radiation therapy alone, the multimodal treatment regiments did not improve either survival or prognosis outcomes [3].

Following the rapid development of imaging techniques and radiotherapy devices, 3D-CRT, IMRT and HT were introduced in quick succession into clinical practice between the 1990s and the beginning of this century. In 2005 the US began to use HT, and in 2007 our hospital installed the first HT treatment unit in China. As compared with conventional IMRT, HT features a wider selection of radiation fields, higher dose to the tumor, and relatively short treatment time. HT is an image-guided radiation therapy (IGRT). Dosimetric coverage of the target with HT is highly conformal and homogeneous. This paper aims to examine the benefits of HT in clinical practice, by reviewing 25 cases receiving HT to treat NKTCL and assessing its efficacy and toxicity.

\section{Materials and Methods}

\section{1) Patient characteristics}

Between August 2008 and April 2013, a total of 25 NKTCL patients were treated with radiotherapy in our department, among those patients who received HT and had medical records that were kept intact. All of them had a KPS score of more than 80 . The clinical characteristics of the patients are summarized in Table 1 . The primary site was the nasal cavity in all the patients. The disease was staged according to the Ann Arbor staging system. Stage IE was subdivided into limited Stage IE (tumor confined to the nasal cavity) and extensive Stage IE (tumor spreading beyond the nasal cavity to neighboring organs or tissues, but no lymph node or distant metastases).

\section{2) Chemotherapy}

In patients treated with HT, three of them had not received chemotherapy, one received concurrent chemoradiation with CHOP plus L-ASP, two received sequential chemotherapy following irradiation, and the others received 1 - 2 cycles of induction chemotherapy followed by irradiation and then sequential chemotherapy. CHOP-L with 1 - 7 cycles (median: 4 cycles) was used as the main chemotherapy regimen.

\section{3) Methodology}

Patients were immobilized in the supine position with a thermoplastic mask. Treatment-planning CT images were acquired from the top of the head to inion with a 3-mm slice thickness. The dose rate was calibrated at 860 cGy/min. The leaf width was $6.25 \mathrm{~mm}$ at isocenter. A $6 \mathrm{MV}$ linear accelerator delivered radiation in a helical mode. The GTV was the gross demonstrable extent and location of the tumor. If the visible tumor disappeared following chemotherapy treatment, the CTV will then be delineated, which included the tumor location and its possible extension pathways (such as nasopharynx, palate and paranasal sinuses). If the tumor was confined to one side of the nasal cavity, and no neighboring organs or tissues were affected, the CTV should include nasal cavities and anterior ethmoid sinuses on both sides; hard palate, as well as maxillary sinus on the same side. If the tumor spread beyond the nasal cavity, the CTV should extend to neighboring organs or tissues which had been affected. Prophylactic neck irradiation was recommended at the same time for patients with a confirmed diagnosis of cervical metastases. Expansion of the CTV by $3 \mathrm{~mm}$ in all directions resulted in the planning target volume (PTV) to account for immobilization inaccuracy. In this study the GTV received TD ranging from 50 to 
Table 1. Clinical characteristics of the study patients.

\begin{tabular}{|c|c|c|}
\hline Characteristics & Cases & $\%$ \\
\hline \multicolumn{3}{|l|}{ Gender } \\
\hline Male & 15 & 60 \\
\hline Female & 10 & 40 \\
\hline \multicolumn{3}{|l|}{ Age } \\
\hline Median & 48 & \\
\hline Range & $22-67$ & \\
\hline \multicolumn{3}{|l|}{ Ann Arbor staging } \\
\hline IE & 11 & 44 \\
\hline IIE & 13 & 52 \\
\hline IVE & 1 & 4 \\
\hline \multicolumn{3}{|l|}{ B symptoms } \\
\hline Yes & 16 & 64 \\
\hline No & 9 & 36 \\
\hline \multicolumn{3}{|l|}{ Modified Ann Arbor staging } \\
\hline Limited stage IE & 8 & 32 \\
\hline Extensive stage IE & 3 & 12 \\
\hline IIE & 13 & 52 \\
\hline IVE & 1 & 4 \\
\hline \multicolumn{3}{|l|}{ Neck lymph node metastasis } \\
\hline Yes & 3 & 12 \\
\hline No & 22 & 88 \\
\hline \multicolumn{3}{|l|}{ Lacto dehydrogenase } \\
\hline Elevated & 2 & 8 \\
\hline Normal & 14 & 56 \\
\hline Unknown & 9 & 36 \\
\hline \multicolumn{3}{|l|}{ IPI index } \\
\hline Low risk (0 - 1 points) & 15 & 60 \\
\hline Low-intermediate risk (2 points) & 1 & 4 \\
\hline High-intermediate risk (3 points) & 1 & 4 \\
\hline High risk (4 - 5 points) & 0 & 0 \\
\hline Unclassified & 8 & 32 \\
\hline
\end{tabular}

56 Gy (median: 50 Gy) at 2 - 2.78 Gy per fraction; and the CTV from 36 to 50 Gy (median: 40 Gy) at 1.6 - 2 Gy per fraction. A dose of $50 \mathrm{~Gy}$ in 25 fractions was prescribed in patients for whom only the CTV was delineated.

4) Efficacy assessment and criteria for adverse reactions

One month after the end of treatment patients were re-examined to assess the efficacy. According to the WHO definitions published in 1979, patients were assigned the four categories of complete response (CR), partial response (PR), stable disease (SD), and progressive disease (PD) on the basis of the measurement criteria. Survival time is defined as the time from the end of radiotherapy to the last follow-up visit or death. The criteria of the Radiation Therapy Oncology Group (RTOG) were used to assess adverse reactions and acute and late toxicity. Acute toxicities include dry mouth, painful swallowing, dysphagia, and skin and mucosa reactions. Late toxicities include dry mouth, taste changes, impaired hearing and vision, and brain injury.

5) Follow-up and statistical analysis

Patients were re-examined at regular intervals at the outpatient clinic after the radiotherapy. Within 2 years 
after the treatment follow-up took place once every 3 months; once every 6 months in between 2 - 5 years; over 5 years then once a year. Telephone follow-ups were conducted from the date when the radiotherapy finished or terminated before 28 August 2013. Follow-up telephone calls enquired about the patient's performance status, time for the latest follow-up and results, whether there were adverse reactions, when the reactions had occurred and extent of the adverse effects. If the patient had died we should enquire about the time and cause of death. The Kaplan-Meier method was used to conduct the survival rate analysis. Results from all cases were analyzed using SPSS ${ }^{\mathrm{TM}}$ (Chicago, IL) Statistics version 13 with a significance level of 0.05 as the basic guidelines for clinical dosimetry significance representation in this study.

\section{Results}

\section{1) Follow-up}

As of $28^{\text {th }}$ August 2013, four patients in the study group were lost to follow-up, who received the last followup call at, respectively, 60, 36, 24, 14 months after radiotherapy. The follow-up rate was $84 \%$. The follow-up lasted 4 - 49 months, with a median follow-up time of 31 months.

\section{2) Dosimetry Analysis}

The total scheduled time from the simulation to the starting treatment was averaged 5 - 6 days (standard deviation 3 - 8 days). From the acquired planning data from the Tomotherapy planning results, total delivery time was reported with 368.4 seconds (Standard deviation $=197.4-614.8$ seconds). The mean pGTV and PTV doses were 53.6 Gy and $49.1 \mathrm{~Gy}$, respectively ( $\mathrm{p}<0.001$ ). All the critical structures were reported within clinical tolerances, and brain stem and spinal cord were reported $37.3 \mathrm{~Gy}$ and $28.8 \mathrm{~Gy}$, respectively ( $<0.001$ ). All lens' doses were less than $5 \mathrm{~Gy}$, and the bi-lateral parotid glands mean doses were $19.7 \mathrm{~Gy}$ and $20.0 \mathrm{~Gy}$, respectively $(\mathrm{p}<0.001)$. All the correlated dose distributions were summarized in Table 2 and Table 3.

\section{3) Efficacy}

One patient terminated before the last 3 treatment sessions due to being unable to tolerate adverse reactions. The others completed radiotherapy as planned. Following the radiotherapy, within 25 patients, thirteen achieved $\mathrm{CR}$, four PR; four had PD; two were lost during follow-up and were not re-examined; two died within one month after radiotherapy. In 21 patients with follow-up records, the overall response (CR + PR) was 81.0\%.

In this group, the 3-year overall survival rate was $87.2 \%$, and the mean survival time was 52.8 months (95\% CI: 45.2 - 60.4 months). As of the last follow-up, the patients in this group had no recurrence or metastasis. A total of three patients died, two resulting from deterioration of health conditions one month after radiotherapy, one resulting from multiple organ failure following bone marrow transplantation at 9 months period. The overall survival rate of 25 patients in the study group was depicted in Figure 1.

\section{4) Side-effects}

During the radiotherapy the blood test results for three patients were lost. Within the other 22 patients, only one had serious bone marrow suppression (Table 4). During radiotherapy process, the acute toxicities mainly involved skin, oropharyngeal mucosa and salivary gland within the radiation field. No patients had acute skin reactions above grade 1 . A few patients had grade 2 salivary gland and oropharyngeal mucosa reactions (Table 5).

Late toxicity is defined as the adverse reactions occurring 6 months after radiotherapy. In nineteen patients who were successfully traced and lived for more than 6 months after radiotherapy, the majority had dry mouth and taste changes in varying degrees, and a small portion of patients had compromised hearing or vision. No brain injury symptoms occurred (Table 6).

\begin{tabular}{ccc|}
\hline \multicolumn{2}{|c|}{ Table 2. Doses delivered to different targets $(\mathrm{Gy})}$. \\
\hline Target & pGTV & PTV \\
\hline Prescription dose & $51.1 \pm 2.1(50.0-56.0)$ & $45.7 \pm 5.1(36.0-50.0)$ \\
Maximum dose & $57.3 \pm 3.0(54.0-64.7)$ & $56.5 \pm 3.58(50.0-66.9)$ \\
Minimum dose & $44.4 \pm 8.2(21.7-54.0)$ & $34.6 \pm 9.93(8.88-48.1)$ \\
Mean dose & $53.6 \pm 2.6(51.5-59.3)$ & $49.1 \pm 4.6(42.1-58.6)$ \\
Volume $\left(\mathrm{cm}^{3}\right)$ & $139.1 \pm 137.8(24.4-535.5)$ & $468.2 \pm 308.8(79.7-1031.6)$ \\
\hline
\end{tabular}


Table 3. Mean dose for organs at risk.

\begin{tabular}{cc}
\hline \multicolumn{1}{c}{ Mean value (Range, Gy) } \\
Brainstem $\mathrm{D}_{\max }$ & $37.3(19.4-57.8)$ \\
Spinal cord $\mathrm{D}_{\max }$ & $28.8(3.7-50.8)$ \\
Left optic nerve & \\
$\mathrm{D}_{\max }$ & $48.1(37.3-63.8)$ \\
$\mathrm{D}_{\operatorname{mean}}$ & $39.8(23.8-56.6)$ \\
Right optic nerve & \\
$\mathrm{D}_{\max }$ & $47.1(34.8-62.9)$ \\
$\mathrm{D}_{\operatorname{mean}}$ & $38.7(26.5-59.2)$ \\
Left eyeball & \\
$\mathrm{D}_{\max }$ & $39.1(21.2-51.3)$ \\
$\mathrm{D}_{\operatorname{mean}}$ & $14.0(5.3-27.7)$ \\
Right eyeball & \\
$\mathrm{D}_{\max }$ & $40.3(20.7-49.9)$ \\
$\mathrm{D}_{\operatorname{mean}}$ & $14.8(7.45-48.5)$ \\
Left lens $\mathrm{D}_{\max }$ & $4.7(2.7-7.8)$ \\
Right lens $\mathrm{D}_{\max }$ & $4.5(2.8-6.6)$ \\
Left parotid glands $\mathrm{D}_{\text {mean }}$ & $19.7(7.0-40.0)$ \\
Right parotid glands $\mathrm{D}_{\text {mean }}$ & $20.0(6.7-38.9)$ \\
Oral cavity $\mathrm{D}_{\text {mean }}$ & $30.2(16.3-45.8)$ \\
\hline
\end{tabular}

Table 4. Bone marrow suppression during irradiation (n, \%).

\begin{tabular}{cccc}
\hline & WBC & Platelet & Anemia \\
\hline Grade 0 & $17(77.3)$ & $19(86.4)$ & $17(77.3)$ \\
Grade 1 & $2(9.1)$ & $1(4.5)$ & $5(22.7)$ \\
Grade 2 & $2(9.1)$ & $2(9.1)$ & 0 \\
Grade 3 & 0 & 0 & 0 \\
Grade 4 & $1(4.5)$ & 0 & 0 \\
\hline
\end{tabular}

Table 5. Acute toxicities (n, \%).

\begin{tabular}{cccc}
\hline & Skin reactions & Salivary gland & Dysphagia \\
\hline Grade 0 & $3(12)$ & $7(28)$ & $3(12)$ \\
Grade 1 & $22(88)$ & $14(56)$ & $18(72)$ \\
Grade 2 & 0 & $4(16)$ & $4(16)$ \\
Grade 3 & 0 & 0 & 0 \\
Grade 4 & 0 & 0 & 0 \\
\hline
\end{tabular}

Table 6. Late toxicities (n, \%).

\begin{tabular}{ccccc}
\hline & Salivary gland & Taste & Vision & Hearing \\
\hline Grade 0 & $9(47.4)$ & $10(52.7)$ & $17(89.4)$ & $16(84.2)$ \\
Grade 1 & $8(42.1)$ & $7(36.8)$ & $1(5.3)$ & $2(10.5)$ \\
Grade 2 & $2(10.5)$ & $2(10.5)$ & $1(5.3)$ & $1(5.3)$ \\
Grade 3 & 0 & 0 & 0 & 0 \\
Grade 4 & 0 & 0 & 0 & 0 \\
\hline
\end{tabular}




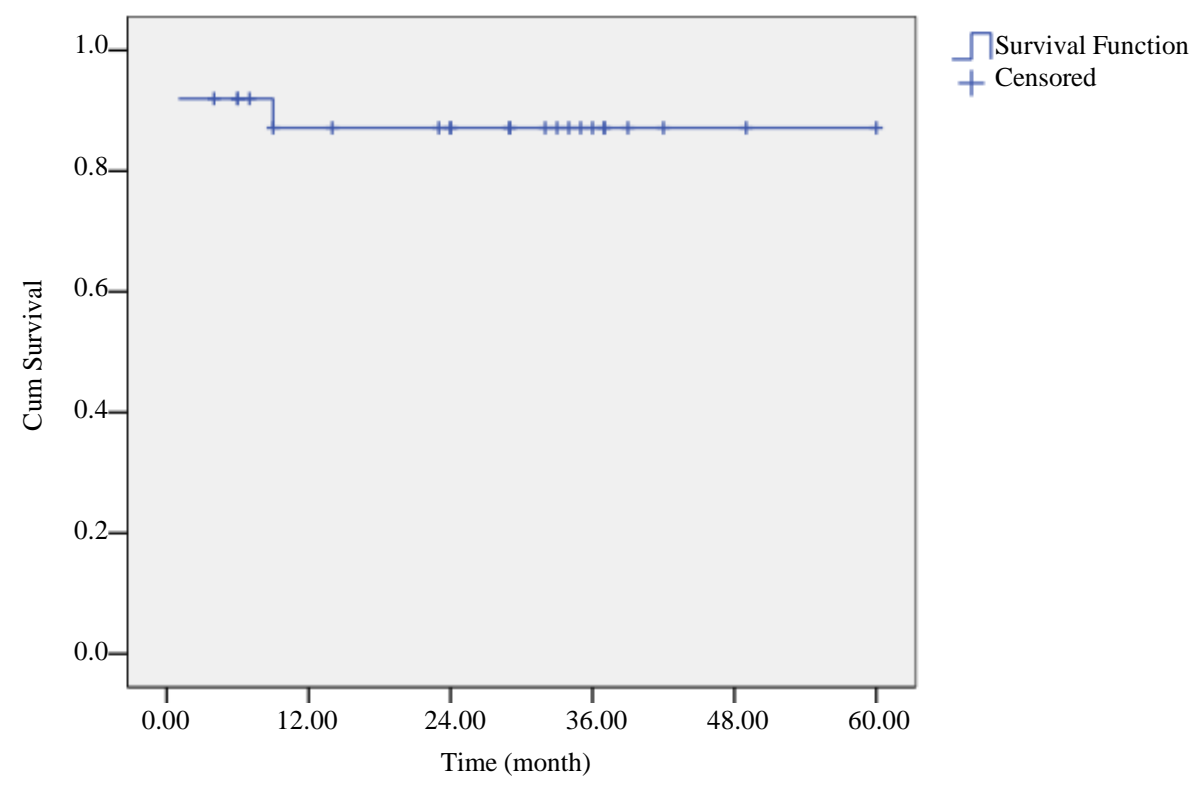

Figure 1. Overall survival rate in 25 patients.

\section{Discussion}

The NKTCL shows a strong association with Epstein-Barr virus. One of the obvious pathological features is the angio-centric distribution of the tumor cells and angio-destruction pattern. Patients usually present with necrotic changes in the nasal cavity. Patients are usually diagnosed with the disease at the localized Stage I - II, and regional lymph node metastases are very rare. Although resistant to chemotherapy, the tumor is sensitive to irradiation. Therefore, radiotherapy is chosen as the main treatment method for early-stage NKTCL. A higher radiation dose and wider radiation field can help improve the local control. NKTCL at the limited Stage IE, if consisting of no negative prognostic indicators, can be treated with radiotherapy alone. Irradiation followed by chemotherapy is recommended for patients at Stage IIE and IE with negative prognostic indicators. Patients with disease above Stage III should use chemotherapy as the main treatment modality, coupled with irradiation at the local site. Many studies have shown that the medians of total radiation doses for NKTCL are 50 - 58 Gy [4]-[6]. A number of large-scale retrospective studies report that a radiation dose of $\geq 50 \mathrm{~Gy}$ can achieve better local control [7] [8]. A study conducted by the Tumor Hospital affiliated to the Chinese Academy of Medical Sciences revealed that the 5-year overall survival rates for patients at Stage I - II range between $46 \%$ and $78 \%$ [1]. Patients with early-stage NKTCL could have long-term survival; therefore, we could be able to achieve higher survival rate while maintaining or improving their quality of life following radiotherapy regiment.

An ideal treatment plan for radiotherapy was to achieve cure by prescribing as high a radiation dose as possible to the target volumes, and simultaneously reduced the impact or damage to healthy surrounding tissues. Side effects of radiation therapy depend on which critical organs were irradiated and the doses were given with respect to the critical dose tolerances. In most of the planning situation, HT possessed better conformal target coverage and more homogeneous dose distribution, with superior dose shaping capability and critical organ sparing compared with conventional 3D/IMRT plans [9]. The radiation is delivered slice-by-slice in a helical or spiral fashion. There has been a dosimetric research article comparing HT and conventional LINAC based IMRT in treating head and neck cancer [10]. In this study, planning results suggested the HT has certain clinical advantage over IMRT from the dosimetric aspects. In another paper, Tomita et al. compared 3D-CRT plans and HT plans from the dosimetric perspective in the treatment of NKTCL, which showed $\mathrm{V}_{95 \%}$ for 3D-CRT and HT was respectively $84.5 \pm 2.7$ and $99.0 \pm 0.9(\mathrm{p}<0.0001)$, was respectively $1.56 \pm 0.33$ and $1.51 \pm 0.20(\mathrm{p}=0.71)$, and homogeneity index (HI) was respectively $0.29 \pm 0.06$ and $0.046 \pm 0.022(\mathrm{p}<0.0001)$ [11]. Those research studies confirmed that in terms of $\mathrm{V}_{95 \%}$ and HI, HT is significantly superior to 3D-CRT in the treatment of NKTCL. Our department conducted a dosimetric study to compare HT and IMRT in the treatment of NKTCL, in which HT showed a clear advantage over IMRT $(\mathrm{p}<0.05)$. The mean CI of HT was 0.81 and mean HI was 1.08; while the mean CI of IMRT was 0.71 and mean HI of 1.12. As for the maximum and mean dose to lens, parotid gland 
and brainstem, HT delivered less radiation than IMRT [12].

There has been limited research work conducted on whether HT's advantage from the dosimetric aspect can be embodied in the treatment of NKTCL. In early-stage NKTCL patients, using radiotherapy only or chemoradiation can achieve a CR rate of 70\% - 94\% [13], while the CR rate following radiotherapy in this study was only $61.9 \%$ (13/21). This may follow from small sample size and incomplete follow-up records. In this group the 3-year overall survival rate was $87.2 \%$, and the mean survival time was 52.8 months (95\% confidence level: 45.2 - 60.4). Similar to this study, Kim et al. [14] has reported the 3-year survival rate with $86 \%$ in patients of Stage I-II NKTCL treated with chemo-radiation. Looking back at the three deaths in this group, two were diagnosed at Stage IIE (induction chemotherapy given with CHOP-L one week before radiotherapy), and one at Stage IVE (no chemotherapy received). One Stage IIE patient had a grade 4 low white blood cell count during radiotherapy, but completed the radiotherapy after treatment leading to an increase in white blood cell count. The patient still expired one month after radiotherapy, due possibly to serious bone marrow suppression and bad health conditions. As for the other one, the anterior lymph nodes in the left neck were affected during radiotherapy. A Dose to Target (DT) of $50 \mathrm{~Gy} / 25 \mathrm{~F}$ was prescribed to nasal cavities on both sides, ethmoid sinuses on both sides, maxillary sinuses on both sides and nasopharynx; and a DT of 50 Gy/20F to the lymphatic drainage system in the left neck. Chemotherapy with 3 cycles of CHOP-L and DICE-L was administered after radiotherapy. The patient had a grade 2 low white blood cell count, grade 1 low platelet count, and grade 1 anemia during radiotherapy. Possibly because of the fact that the disease had not been controlled after chemo-radiation, the patient expired 9 months after radiotherapy due to multiple organ failure when receiving bone marrow transplantation. As for the Stage IVE patient, who was of older age (at 65 years old), liver metastases had been present when this patient received the final diagnosis. We had planned to prescribe a DT of $50 \mathrm{~Gy} / 25 \mathrm{~F}$, but the patient had grade 2 dry mouth and pharyngeal pain during radiotherapy. Therefore, patient was quitting the last 3 sessions of radiotherapy due to poor physical condition which was unable to tolerate the radiation reactions. Eventually, the patient expired one month after radiotherapy as a result of persistent low-grade fever and multiple organ failure. As of the last follow-up, patients in this group had no recurrence or metastasis. With all the follow-up analysis, for this group of clinical cases, we can be seen HT provides good efficacy in the treatment of NKTCL.

As for evaluation of acute toxicities, only one patient had a grade 4 low white blood cell count during radiotherapy, while no bone marrow suppression above grade 2 occurred in the other patients. On the other hand, Yamaguchi et al. treated 33 NKTCL patients with 3D-CRT coupled with combination chemotherapy, and found there were low counts of white blood cell, platelet and hemoglobin at grade 3 or 4 [15]. In terms of both number of patients suffering from side effects and the extent, the patients receiving HT in this study yielded more favorable results. Acute toxicities mainly include skin reactions, dry mouth and pharyngeal pain. In this study, no patients had skin reactions above grade 1; whereas in Wang et al.'s study, where NKTCL patients received IMRT (DT $50 \mathrm{~Gy} / 25 \mathrm{~F}$ prescribed to PTV, an additional 5 - $10 \mathrm{~Gy}$ to visible tumor), 16.7\% of patients had grade 2 acute skin reactions [16]. In this study, similar to Wang et al.'s (16.7\%), 16\% of patients had grade 2 dry mouth; but there were more grade 0 dry mouth patients (28\%) than in Wang et al.'s study (16.7\%). No patients had dysphagia above grade 2, and there was one case in Wang et al.'s study having grade 3 dysphagia; moreover, there were fewer patients with grade 2 dysphagia in this study (16\% vs. 26.2\%) [16]. As can be seen from above, in comparison with IMRT HT, has a better safety profile to some extent in terms of extent and occurrence of acute toxicities.

Late toxicity is defined as the adverse reactions occurring at 6 months after the end of radiotherapy. In this study the most common late toxicities include dry mouth and taste changes. Zheng et al. conducted a study about the efficacy and safety of IMRT in treating NKTCL patients, in which there were fewer patients not presenting with dry mouth than in this study (42.9\% vs. 47.4\%), and more patients with grade 1 and 2 dry mouth symptom (42.9\% vs. $42.1 \%, 14.3 \%$ vs. 10.5\%). As for taste changes, there were fewer patients not presenting with this reaction than in this study (42.9\% vs. $52.7 \%$ ), and more patients with grade 1 taste changes (50.0\% vs. 36.8\%); however, Zheng et al.’s study had fewer patients with grade 2 taste changes (7.1\% vs.10.5) [6].

\section{Conclusions}

From this first clinical systematic study, our research results showed that there were very few clinical subjects which experienced compromised hearing and vision loss at late stage. In summary, only 2 patients had impaired vision (grade 1: 1 case; grade 2: 1 case), and 3 had impaired hearing (grade 1: 2 cases; grade 2: 1 case). No severe 
late reactions (above grade 2) had occurred. As a whole, HT has, in general, a better safety profile in terms of occurrence of late toxicities resulting from radiotherapy to treat NKTCL patients in our follow-up cases.

However, the direct comparison of HT and IMRT in terms of the above mentioned variables depends on future research and more clinical data with long term analysis. Results generated from this study can provide a basis for choice of treatment options in routine clinical practice. With current follow-up trend in our clinic, we hope that HT dosimetric benefits could eventually translated into more NKTCL patient survival with good quality of life index.

\section{Acknowledgements}

We are very grateful to the patients and their families for participating in this study. We also thank all of the clinicians, nurses, pathologists and study coordinators for their contributions to the work.

\section{References}

[1] Aozasa, K., Takakuwa, T., Hongyo, T. and Yang, W. (2008) Nasal NK/T-Cell Lymphoma: Epidemiology and Pathogenesis. International Journal of Hematology, 87, 110-117. http://dx.doi.org/10.1007/s12185-008-0021-7

[2] Yin, W., Yu, Z., Xu, F. and Hu, Y. (2008) Clinical Radiation Oncology. 4th Edition, China Union Medical University Press, Beijing, 754-764.

[3] Li Y., Liu, Q., Wang, W., Jin, J., Song, Y., Wang, S., Liu, Y., Zhou, L. and Yu, Z. (2011) Failure Patterns and Clinical Implications in Early Stage Nasal Natural Killer/T-Cell Lymphoma Treated with Primary Radiotherapy. Cancer, 117, 5203-5211. http://dx.doi.org/10.1002/cncr.26167

[4] Li, Y., Wang, H., Jin, J., Wang, W., Liu, Q., Song, Y., Wang, Z., Qi, S., Wang, S., Liu, Y. and Yu, Z. (2012) Radiotherapy Alone with Curative Intent in Patients with Stage I Extranodal Nasal-Type NK/T-Cell Lymphoma. International Journal of Radiation Oncology • Biology • Physics, 82, 1809-1815. http://dx.doi.org/10.1016/j.jjrobp.2010.10.040

[5] Yong, Y., Zhang, Y. and Bin, L. (2009) Radiotherapy in Early Nasal Type NK/T Cells in Lymphoid Tumor Effect of the Combined Treatment and Prognosis. Chinese Journal of Radiation Oncology, 18, 285-289.

[6] Zheng, L., Ma, X., Wang, J., Xu, Q., Zhang, S. and Hu, J. (2013) IMRT with Conventional Radiotherapy in Early Stage Nasal NK/T-Cell Lymphoma Patients Treated Controls. China Oncology, 23, 229-234.

[7] Li, Y., Yao, B., Jin, J., Wang, W., Liu, Y., Song, Y., Wang, S., Liu, X., Zhou, L., He, X., Lu, N. and Yu, Z. (2006) Radiotherapy As Primary Treatment for Stage IE and IIE Nasal Natural Killer/T-Cell Lymphoma. Journal of Clinical Oncology, 24, 181-189. http://dx.doi.org/10.1200/JCO.2005.03.2573

[8] Ma, H., Qian, L., Pan, H., Yang, L., Zhang, H., Wang, Z., Ma, J., Zhao, Y., Gao, J. and Wu, A. (2010) Treatment Outcome of Radiotherapy Alone versus Radiochemotherapy in Early Stage Nasal Natural Killer/T-Cell Lymphoma. Medical Oncology, 27, 798-806. http://dx.doi.org/10.1007/s12032-009-9288-7

[9] Xu, S., Wang, L., Dai, X., Huang, H. and Xie, C. (2008) Helical Tomotherapy System Theory and Its Application Principle and Application of Helical Tomotherapy. Chinese medical Equipment Journal, 29, 100-102.

[10] Cui, D., Dai, K., Ma, L., Xu, S., Wang, S., Xia, Z., Chun, F. and Lam, S. (2008) Nasopharyngeal Helical Tomotherapy IMRT and Conventional Accelerators Dosimetric Comparison: A Dosimetric Comparison between Helical Tomotherapy and Linear Accelerator-Based Intensity Modulated Radiation Therapy for Nasopharyngeal Carcinoma. Chinese Journal of Radiation Oncology, 5, 169-173.

[11] Tomita, N., Kodaira, T., Tachibana, H., Nakamura, T., Nakahara, R., Inokuchi, H., Mizoquchi, N. and Takada, A. (2009) A Comparison of Radiation Treatment Plans Using IMRT with Helical Tomotherapy and 3D Conformal Radiotherapy for Nasal Natural Killer/T-Cell Lymphoma. The British Journal of Radiology, 82, 756-763. http://dx.doi.org/10.1259/bjr/83758373

[12] Gong, H., Xie, C. and Xu, S. (2011) Dosimetric Comparison between Helical Tomotherapy and Step-and-Shoot Intensity Modulated Radiation for Nasal T/NK-Cell Lymphoma. The Practical Journal of Cancer, 4, 377-380.

[13] Wu, R.J., Li, Y.X., Jin, J., Wang, S.L., Yue, P., Song, Y.W., Ren, H., Liu, Q.F., Wang, C.Y., Qi, S.N., Lu, N.N., Bo, E.P., Zhou, S. and Yu, Z.H. (2011) Webster Ring Primary Diffuse Large B-Cell and Extranodal Nasal Type NK Compare Clinical Features and Prognosis/T-Cell Lymphoma. Chinese Journal of Radiation Oncology, 20, 80-88.

[14] Kim, S., Kim, K., Kim, B., Kim, C., Suh, C., Huh, J., Lee, S., Kim, J., Cho, J., Lee, G., Kang, K., Eom, H., Pyo, H., Ahn, Y., Ko, Y. and Kim, W. (2009) Phase II Trial of Concurrent Radiation and Weekly Cisplatin Followed by VIPD Chemotherapy in Newly Diagnosed, Stage IE to IIE, Nasal, Extranodal NK/T-Cell Lymphoma: Consortium for Improving Survival of Lymphoma Study. Journal of Clinical Oncology, 27, 6027-6032.

http://dx.doi.org/10.1200/JCO.2009.23.8592 
[15] Yamaguchi, M., Tobinai, K., Oguchi, M., Ishizuka, N., Kobayashi, Y., Isobe, Y., Ishizawa, K., Maseki, N., Toh, K., Usui, N., Wasada, I., Kinoshita, T., Ohshima, K., Matsuno, Y., Terauchi, T., Nawano, S., Ishikura, S., Kagami, Y., Hotta, T. and Oshimi, K. (2009) Phase I/II Study of Concurrent Chemoradiotherapy for Localized Nasal Natural Killer/ T-Cell Lymphoma: Japan Clinical Oncology Group Study JCOG0211. Journal of Clinical Oncology, 27, 5594-5600.

[16] Wang, H., Li, Y., Wang, W., Jin, J., Dai, J., Wang, S., Liu, Y., Song, Y., Wang, Z., Liu, Q., Fang, H., Qi, S., Liu, X. and $\mathrm{Yu}, \mathrm{Z}$. (2012) Mild Toxicity and Favorable Prognosis of High-Dose and Extended Involved-Field IntensityModulated Radiotherapy for Patients with Early-Stage Nasal NK/T-Cell Lymphoma. The British Journal of Radiology, 82, 1115-1121. http://dx.doi.org/10.1016/j.ijrobp.2011.02.039 
Scientific Research Publishing (SCIRP) is one of the largest Open Access journal publishers. It is currently publishing more than 200 open access, online, peer-reviewed journals covering a wide range of academic disciplines. SCIRP serves the worldwide academic communities and contributes to the progress and application of science with its publication.

Other selected journals from SCIRP are listed as below. Submit your manuscript to us via either submit@scirp.org or Online Submission Portal.
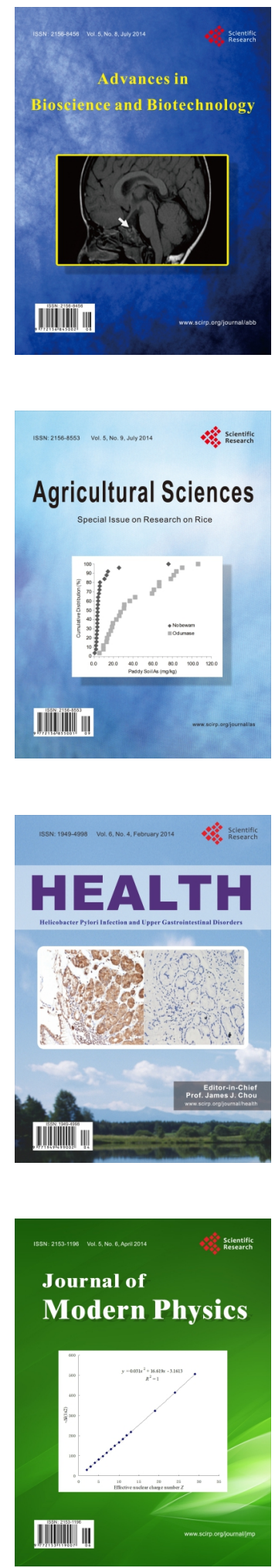
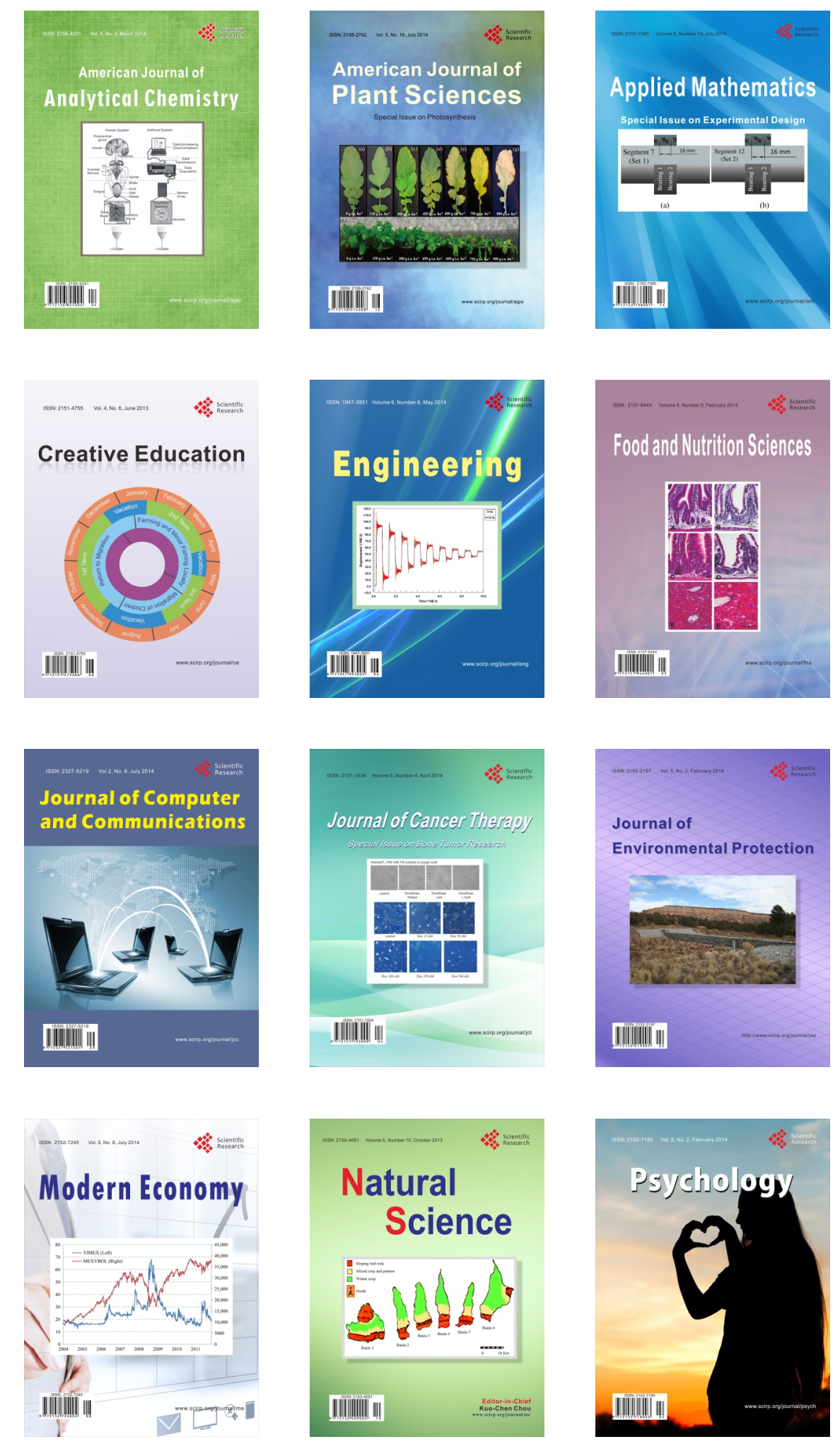